\title{
Messengers of hope
}

\author{
Emergency Use Authorizations for two mRNA COVID-19 vaccines represent a turning point in the pandemic.
} They also herald a new era for vaccinology.

O ne year ago, few would have tipped mRNA vaccines as the world's best hope. They had been tested in a handful of human trials. They had been injected into fewer than 1,800 people. And efficacy data in humans were both sparse and underwhelming. Twelve months on, $\sim 34,000$ people have received mRNA immunizations in human trials of two mRNA products targeting SARS-CoV-2 spike (S) protein. Both products provide impressive efficacy, notably in individuals 65 or older. Emergency Use Authorizations of BioNTech/Pfizer's BNT162b2 and Moderna's mRNA-1273 vaccines raise hopes that the pandemic could finally turn a corner. They may even presage an era where vaccines are designed in computers, iteratively optimized in discovery and manufactured on demand - all without expensive and finicky cell culture.

The new nucleoside-modified mRNA vaccines are chemicals that have been almost fully disclosed. They incorporate a trinucleotide cap 1 analog $\left(\left(\mathrm{m}_{2}{ }^{73^{\prime}-O}\right) \mathrm{Gppp}\left(\mathrm{m}^{2^{\prime}-O}\right)\right.$ ApG), contain $N^{1}$-methylpseudouridine instead of uridine, and encode an optimized (P2-mutated) full-length S glycoprotein encapsulated in lipid nanoparticles (LNPs) containing polyethylene glycol and cholesterol. The BNT162b2 LNP also contains (4-hydroxybutyl)azanediyl) bis(hexane-6,1-diyl)bis(2-hexyldecanoate) and 1,2-distearoyl-sn-glycero3-phosphocholine, whereas Moderna's LNP contains SM-102 (most likely heptadecan-9-yl 8-((2-hydroxyethyl) (6-oxo-6-(undecyloxy)hexyl)amino) octanoate) and 1,2-dimyristoyl-rac-glycero3-methoxypolyethylene glycol-2000 (PEG). The BioNTech vaccine is administered intramuscularly (IM) as two $30-\mu \mathrm{g}$ doses 21 days apart, the Moderna product as two $100-\mu \mathrm{g}$ IM doses 28 days apart.

Two other types of synthetic mRNA LNP COVID-19 vaccines have also entered testing: CureVac just initiated a phase $2 / 3$ trial for its unmodified uridine-deficient mRNA product $(\mathrm{CVnCoV})$, and during the summer Arcturus Therapeutics and Imperial College London commenced phase 1 testing of self-amplifying RNA vaccines based on an alphavirus that encode non-structural proteins to replicate the S-protein immunogen.

The BioNTech and Moderna vaccines work spectacularly. Of the 20,033 people who received $\mathrm{BNT} 162 \mathrm{~b} 2$, efficacy outcomes one week after the second dose were $95 \%$, a level consistent $(\geq 93 \%)$ across all demographic subgroups. In the 13,218 participants receiving two doses of Moderna's vaccine, efficacy was $93.4 \%$ for people 18 to 64 years old and $86.4 \%$ for those ages 65 and up. For both products, adverse events elevated in the vaccine arm of the trial included fatigue, fever, headache, myalgia, arthralgia, pain/swelling at the injection site, lymphadenopathy, nausea, erythema, Bell's palsy and appendicitis.

This triumph for RNA vaccine development has been three decades in the making. The first reports that foreign mRNA could express protein and induce cellular immunity when injected into mouse muscle came in the 1990s. Until the early noughts, however, progress was dogged by inflammatory reactions mediated by innate immune sensors for double-stranded (ds)RNA (for example, cytoplasmic protein kinase $\mathrm{R}$ (PKR) and $2^{\prime}-5^{\prime}$-oligoadenylate synthetase, IFIT1, MDA5 and RIG-I) and for single-stranded RNA (for example, endosomal Toll-like receptor 3 (TLR3), TLR7 and TLR8). This reactogenicity, together with mRNA's fragility (to RNases), large size (thousands of bases) and mediocre potency, led to widespread skepticism around the modality.

A breakthrough came in 2005 when Katalin Karikó, Drew Weissman and their colleagues demonstrated that nucleoside modifications, such as pseudouridine and 5-methylcytidine, ablate TLR signaling (and later when they showed similar dampening of PKR activation). That modified RNA actually produces more protein than unmodified mRNA was a big surprise. Reactogenicity was addressed via codon optimization and removal of dsRNA contaminant from the in vitro transcription reaction by various purification methods.

Immune reactivity was not the only problem, however. As with other nucleic acid modalities, mRNAs are large molecules to get into cells - either via ex vivo delivery to cells (for example, as in dendritic cell cancer vaccines) or direct parenteral injection with or without a carrier. Many carriers were tried, including protamine, cationic liposomes, cationic nanoemulsions, dendrimers, polymers and LNPs. In 2012, Tekmira Pharmaceuticals' ionizable LNPs, which galvanized Alnylam's siRNA programs, began testing for mRNA; the same year, Novartis vaccines (now part of GlaxoSmithKline) showed that ionizable LNPs could potentiate a self-amplifying RNA vaccine. Today, ionizable LNPs are the carrier of choice.

Over the years, mRNA stability and translation efficiency has also been tuned by optimizing regulatory elements in the $5^{\prime}$ and $3^{\prime}$ untranslated regions, poly (A) tail length and codon usage in the construct. Similarly, mRNA-LNP formulation is moving from macroscale mixing to more reproducible microfluidic mixing devices.

Unlike DNA vaccines, mRNA vaccines do not have to cross the nuclear envelope, they pose no risk of genomic integration, and they work in both dividing and non-dividing cells. Unlike protein and peptide vaccines, they are not restricted by major histocompatibility complex haplotype. That said, they have burdensome cold-chain requirements and are currently the most expensive COVID-19 vaccines.

What is perhaps most exciting is the potential for mRNA as a rapid and generic platform for any desired immunogen(s). As upstream computational design and downstream processing and manufacture are standardized, custom work will mostly involve optimizing specific mRNA constructs to express efficiently in cells of interest. For SARS-CoV-2, Moderna was able to create mRNA-1273 in just six weeks after disclosure of the draft viral genome - a record turnaround for a vaccine candidate. For future pandemics, vaccine templates could even be prepared in advance for more rapid deployment.

By January 2022, we will know whether the promise is realized. By then, millions of people will have received these vaccines. Pooled data from VAERS, Sentinel and other initiatives will provide a clearer picture of rare serious adverse events associated with immunization, such as severe allergic reactions to PEG. And burning issues beyond safety - whether these products can be used in pregnant women or children (given multisystem inflammatory syndrome), whether they afford long-term protection and whether asymptomatic vaccinated individuals are capable of transmission - will be answered.

For now, though, these vaccines represent a new hope: the beginning of the end for this pandemic - and the advent of a new era in vaccinology.

Published online: 29 December 2020 https://doi.org/10.1038/s41587-020-00807-1 